\title{
EDIFICATE
}

I Congreso de Escuelas de Edificación y Arquitectura Técnica de España

València, 4 y 5 de noviembre de 2021

Escuela Técnica Superior de Ingeniería de Edificación

Universitat Politècnica de València

Doi: https://doi.org/10.4995/EDIFICATE2021.2021.13513

\section{Equipo docente para la Docencia Digital y la Coordinación Transversal de las asignaturas del Módulo de Gestión del Proceso del Grado de Edificación. Universidad de Granada}

\section{Teaching Team for Digital Teaching and Transversal Coordination of the subjects of the Process Management Module of the Degree in Building. University of Granada}

\begin{abstract}
Antonio J. Aguilar ${ }^{\mathrm{a}}$, Carlos Benavides de la Fuente ${ }^{\mathrm{b}}$ Gloria Cuenca-Moyanoc ${ }^{\mathrm{c}}$, Lourdes Gutiérrez-Carrillo $^{d}$, María L. de la Hoz-Torres ${ }^{e}$, María Martín-Morales ${ }^{f}$, M. Dolores Martínez-Aires $^{\mathrm{g}}$, Manuel Martínez Carrillo $^{\mathrm{h}}$ y Raquel Nieto-Álvarez

aDpto. Física Aplicada, Universidad de Granada (UGR), antojes@ugr.es, 'Estudiante del Grado de Edficación (UGR), carlosbf@correo.ugr.es, 'Dpto. Construcciones Arquitectónicas (DCA) (UGR), gloriacuenca@ugr.es, dDCA (UGR), mlgutier@ugr.es, eDpto. Física Aplicada (UGR), mlhoz@ugr.es,

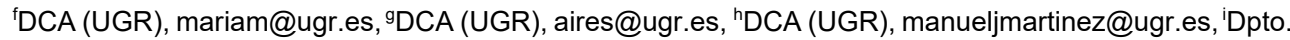
Expresión Gráfica Arquitectónica y en la Ingeniería, rnieto@ugr.es
\end{abstract}

\begin{abstract}
The healthcare crisis caused by COVID-19 has had an enormous impact on the development of academic activity, requiring the adaptation of traditional teaching methodology (mainly face-to-face) to blended or virtual scenarios. These circumstances have highlighted the need to implement new methodologies and tools, as well as the use of different platforms which are not acquainted for the teaching staff.

In this context, the Teaching Team for Digital Teaching and Transversal Coordination on the subjects of the Process Management Module of the Degree in Building at the University of Granada has been created. The objective of the project has been to empower the teaching staff through the training in digital tools that improve the available resources for the development of teaching in an online scenario; in adition to coordinating the process between different subjects, improving the teaching-learning experience and making the subject more attractive for the students.
\end{abstract}

This paper presents the specific objectives set, the structure of the sessions, and the methodology and materials used, which have helped the participating 
teachers to develop fundamental digital competences for the implementation of new teaching tools and methodologies.

Keywords: teaching team, digital training, project management model, BIM, advanced Moodle tools, good teaching practices

\section{Resumen}

La crisis sanitaria provocada por la COVID-19 ha tenido un enorme impacto en el desarrollo de la actividad académica, requiriendo la adaptación de la metodología de docencia tradicional (principalmente presencial) a escenarios semipresenciales o virtuales. Estas circunstancias han puesto de manifiesto la necesidad de implementar nuevas metodologías y herramientas, así como el uso de distintas plataformas con las que el profesorado no está familiarizado.

En este contexto se ha creado el Equipo docente para la Docencia Digital y la Coordinación Transversal de las asignaturas del Módulo de Gestión del Proceso del Grado de Edificación de la Universidad de Granada. El objetivo ha sido capacitar al profesorado a través de formación en herramientas digitales que permiten mejorar los recursos disponibles para el desarrollo de la docencia en un escenario online, coordinar el proceso entre diferentes asignaturas, mejorando la experiencia de enseñanza-aprendizaje y haciendo más atractiva la asignatura al alumnado.

En este trabajo se presenta los objetivos específicos planteados, las estructura de las sesiones, metodología y materiales utilizados, que han contribuido a que el profesorado participante desarrolle competencias digitales fundamentales para la implementación de nuevas herramientas y metodologías docentes.

Palabras clave: equipo docente, capacitación digital, modelo de gestión de proyectos, BIM, herramientas avanzadas Moodle, buenas prácticas docentes 
Antonio J. Aguilar, Carlos Benavides de la Fuente, Gloria Cuenca-Moyano, Lourdes Gutiérrez-Carrillo, María L. de la Hoz-Torres, María Martín-Morales, M. Dolores Martínez-Aires, Manuel Martínez Carrillo y Raquel Nieto-Álvarez

\section{Introducción}

La pandemia de la COVID-19 ha tenido una gran impacto en todos los sectores, tanto económico como social (Sarkodie \& Owusu, 2021). La docencia universitaria no se ha mantenido ajena, requiriendo de un gran esfuezo de adaptación de las metodologías de docencia tradicional (principalmente presencial) a escenarios semipresenciales o virtuales. En los últimos años, la Universidad de Granada (UGR), principalmente a través de sus Planes de Formación e Innovación Docente (FIDO) (UGR, 2016), ha formentado la creación de los equipos docentes multidisciplinares de formación continua del profesorado como herramienta para favorecer la colaboración entre profesorado experto y principiante, con la mejora continua de la práctica docente, el intercambio de experiencias y el establecimiento de compromisos.

La urgente necesidad de enseñanza online creada por la situación sanitaria, ha hecho que se hayan diseñado un amplio abanico de programas de formación para el profesorado, cursos intensivos, webinar, etc. (UGR, 2020a), con el fin de facilitar el proceso de adaptación, ayudando y acompañando en la difícil tarea de mantener una docencia y atención al alumnado con los estándares de calidad deseados.

Dentro de la estrategias de formación de la UGR se engloba el programa de presentación de solicitudes para la I Convocatoria de Formación de Equipos Docentes para la Docencia Digital con los siguientes objtivos (UGR, 2020b):

- $\quad$ el desarrollo de competencias docentes digitales,

- la implementación de metodologías activas y participativas a través de las plataformas digitales para la docencia,

- la incorporación y el uso didáctico de herramientas y plataformas digitales en los procesos de seguimiento, orientación, tutorización y evaluación del estudiantado,

- la diversificación de los procedimientos de evaluación continua y final mediante herramientas y plataformas digitales,

- $\quad$ el diseño de materiales o recursos docentes digitales, y

- $\quad$ el diseño y la puesta en marcha de cualquier iniciativa de innovación docente ligada a la digitalización de la docencia.

Englobado en esta convocatoria se ha creado el grupo docente denominado Equipo docente para la Docencia Digital y la Coordinación Transversal de las asignaturas del Módulo de Gestión del Proceso del Grado de Edificación. La acción formativa se definió tras el análisis de las herramientas disponibles para el profesorado diseñadas por el Centro de Producción de Recursos para la Universidad Digital (CEPRUD) de la UGR (CEPRUD, 2021), con el objetivo de no repetir contenidos generales, centrándose en herramientas avanzadas.

Por ello, se ha optado por el uso de herramientas digitales específicas para las asignaturas del módulo y, en general, del Grado de Edificación. Todas ellas tienen el objetivo de acercar al alumnado a las obras y profundizar en conceptos curriculares, destacando la creación de recursos digitales interactivos, el uso de la metodología Building Information Modelling (BIM) y la realidad virtual y videos $360^{\circ}$. Además, se han incorporado sesiones para mostrar 
estrategias para asistencia activa y buenas prácticas docentes, usando, entre otras herramientas, Moodle como plataforma para la gestión de aprendizaje.

\section{Objetivo}

En este trabajo se presenta la inciativa de un grupo de profesores del Grado de Edificación de la UGR para crear un Equipo docente para la Docencia Digital y la Coordinación Transversal de las asignaturas del Módulo de Gestión del Proceso del Grado de Edificación.

Se presentarán los objetivos propuestos, la estructura de la acción formativa y los resultados obtenidos.

\section{Desarrollo de la innovación docente}

\subsection{Objetivos del Equipo docente}

El Módulo Gestión del Proceso incluye cinco asignaturas:

- Organización y Programación en Edificación (asignatura obligatoria de 3er curso)

- Gestión de la Calidad (asignatura obligatoria de $4^{\circ}$ curso)

- Prevención y Seguridad (asignatura obligatoria de $4^{\circ}$ curso)

- Proyecto de Gestión del Proceso y Equipos de Obras (asignatura obligatoria de $4^{\circ}$ curso)

- $\quad$ Gestor de proyectos, de producción y de mantenimiento (asignatura optativa de $4^{\circ}$ curso)

El objetivo principal del equipo docente ha sido capacitar al profesorado de dicho módulo en herramientas digitales que permitan mejorar los recursos disponibles para el desarrollo de la docencia en un escenario online, coordinar el proceso entre diferentes asignaturas y mejorar la experiencia de enseñanza-aprendizaje.

Los objetivos específicos del equipo docente han sido los siguientes:

- $\quad$ 01. Coordinar las asignaturas del Módulo Gestión del Proceso en la adaptación a un escenario semipresencial, así como las necesidades en otras asignaturas del los departamentos con más docencia en el grado: Construcciones Arquitectónicas y Expresión Gráfica Arquitectónicas y en la Ingeniería.

- O2. Dotar al profesorado de nuevas herramientas para el diseño de nuevos materiales didácticos digitales y recursos docentes.

- O3. Proporcionar recursos que faciliten el seguimiento y tutorización del estudiantado. 
Antonio J. Aguilar, Carlos Benavides de la Fuente, Gloria Cuenca-Moyano, Lourdes Gutiérrez-Carrillo, María L. de la Hoz-Torres, María Martín-Morales, M. Dolores Martínez-Aires, Manuel Martínez Carrillo y Raquel Nieto-Álvarez

También se han analizado las necesidades de coordinación en el uso de herramientas docentes con otras asignaturas del grado.

\subsection{Componentes}

El equipo docente ha contado con once componentes. Dado que la convocatoria permitía la inclusión de estudiantes, se ha contado con la participación de dos alumnos de cuarto curso. El profesorado incialmente era el siguiente:

- Seis docentes adscritos al Módulo de Gestión de Proyectos

- La Directora de Dpto. de Construcciones Arquitectónicas

- La Secretaria del Dpto. de Expresión Gráfica Arquitectónicas y en la Ingeniería

A este grupo, dado que se envió la infomación a todo el profesorado del Grado de Edificación, se unieron dos nuevas integrantes pertenecientes al Dpto. de Construcciones Arquitectónicas.

Por otro lado, distinto profesorado se ha ido conectando puntualmente a alguna de las sesiones programadas.

\subsection{Estructura}

Para alcanzar los objetivos definidos, se han planificado sesiones agrupadas en tres módulos:

Módulo A. Coordinación de la adaptación de la docencia en los escenarios semipresencial y virtual de las asignaturas del Módulo de Gestión del Proceso, así como con otras asignaturas del Dpto. Construcciones Arquitectónicas y del Dpto. de Expresión Gráfica Arquitectónica y en la Ingeniería. Debate sobre las competencias, herramientas y recursos necesarios para la docencia digital en ambos escenarios. Las sesiones del Módulo A contribuyen a alcanzar el objetivo 01.

Módulo B. Herramientas de creación de contenidos digitales interactivos (desarrollo de contenido web, software Wimba Create, modelos digitales 3D interactivos, metodología Building Information Modelling aplicada a la docencia, Videos $360^{\circ}$, etc.) Las sesiones del Módulo B contribuyen a alcanzar el objetivo $\mathrm{O} 2$.

Módulo C. Herramientas digitales para el seguimiento, planificación y tutorización en la docencia digital (uso de software y plataformas: Kaltura, Miro, Prado, Kahoot, Telegram, etc.) Las sesiones del Módulo C contribuyen a alcanzar el objetivo O3.

\subsection{Metodología, planificación y materiales}

Las sesiones se han estructurado en tres módulos (A, B y C) que desarrollan los objetivos O1, O2 y O3 respectivamente, además de una sesión inicial ( $\mathrm{SI}$ ) y una final (SF). Todos los contenidos se planificaron para ser trabajados en quince sesiones según se recoge en la Tabla 1. 
Cada sesión ha tenido una duración de dos horas: una teórica y la otra de tipo Taller. Al final de cada sesión se ha planificado quince minutos de debate. Esto ha permitido exponer dudas relacionadas con la sesión y otros problemas identificados al implementar recursos digitales en el aula. Se ha fomentado la participación activa, con la utilización de distintas herramientas (por ejemplo, Kahoot, Miro, etc.) y con el diseño de distintas actividades que debían realizarse entre las sesiones (normalmente una semana).

Tabla 1. Contenidos de las sesiones

\begin{tabular}{|c|c|}
\hline $\begin{array}{l}\mathrm{N}^{\circ} \\
\text { Taller }\end{array}$ & Resumen de la sesión \\
\hline SI & $\begin{array}{l}\text { Sesión Inicial de constitución del equipo docente. Presentación del equipo } \\
\text { docente. Planificación y organización de sesiones. }\end{array}$ \\
\hline A3 & $\begin{array}{l}\text { Análisis de las necesidades y competencias transversales de las asignaturas } \\
\text { del Módulo de Gestión de Proyectos con las adquiridas en otras asignaturas } \\
\text { del Departamento de Expresión Gráfica Arquitectónica y en la Ingeniería. }\end{array}$ \\
\hline A1 & $\begin{array}{l}\text { Herramientas del estudiantado durante la docencia virtual: antecedentes, } \\
\text { plataformas y aplicaciones. }\end{array}$ \\
\hline A2 & $\begin{array}{l}\text { Sesión de coordinación transversal de asignaturas del Módulo Gestión del } \\
\text { Proceso del grado Ingeniería en Edificación. Participación de todo el equipo } \\
\text { docente. Creación de Matriz DAFO }\end{array}$ \\
\hline B1 & $\begin{array}{l}\text { Creación de recursos digitales. Introducción: Herramientas de desarrollo de } \\
\text { contenidos digitales: software Wimbra Create. }\end{array}$ \\
\hline B2 & Creación de recursos digitales. Integración en la plataforma PRADO. \\
\hline B3 & $\begin{array}{l}\text { Uso de la metodología Building Information Modelling (BIM) para la mejora de } \\
\text { la docencia. Introducción. }\end{array}$ \\
\hline B4 & $\begin{array}{l}\text { Uso de la metodología Building Information Modelling (BIM) para la mejora de } \\
\text { la docencia. Ejemplo de desarrollo de modelos. }\end{array}$ \\
\hline B5 & $\begin{array}{l}\text { Uso de la metodología Building Information Modelling (BIM) para la mejora de } \\
\text { la docencia. Integración en la plataforma de docencia: Sketchfab e Integración } \\
\text { en la plataforma PRADO. }\end{array}$ \\
\hline B6 & $\begin{array}{l}\text { Uso de realidad virtual y videos } 360^{\circ} \text { para el desarrollo de prácticas en un } \\
\text { escenario no presencial. }\end{array}$ \\
\hline B7 & $\begin{array}{l}\text { Creación de recursos digitales. Integración en la plataforma PRADO: Recursos } \\
\text { interactivos digitales H5P. }\end{array}$ \\
\hline $\mathrm{C} 1$ & $\begin{array}{l}\text { 1.- Herramientas digitales para la Asistencia activa. } \\
\text { 2.- Kaltura como herramienta de grabación y edición de contenido digital. }\end{array}$ \\
\hline $\mathrm{C} 2$ & $\begin{array}{l}\text { Autoevaluación y evaluación por pares del trabajo del alumnado: uso de la } \\
\text { herramienta TALLER de PRADO }\end{array}$ \\
\hline C3 & $\begin{array}{l}\text { Uso de plataformas digitales para el seguimiento y comunicación con el } \\
\text { alumnado: Telegram. }\end{array}$ \\
\hline
\end{tabular}


Antonio J. Aguilar, Carlos Benavides de la Fuente, Gloria Cuenca-Moyano, Lourdes Gutiérrez-Carrillo, María L. de la Hoz-Torres, María Martín-Morales, M. Dolores Martínez-Aires, Manuel Martínez Carrillo y Raquel Nieto-Álvarez

SF Sesión Final y cierre del equipo docente. Definición de puntos fuertes y débiles del proyecto docente. Definición de futuras acciones.

Dado que la UGR cuenta con un acuerdo con Google Apps, en el proyecto se ha hecho uso de las distintas Apps, algunas poco utilizadas por el profesorado. Además, el uso de estas herramientas ha permitido mostrar las ventajas de su implementación en la actividad docente, así como su potencial. Se han utilizado las siguientes:

- Google Meet

- Google Drive

- Google Site

- Google Formulario

- Google Calendar

- Google Doodle

Las sesiones se han realizado a través de Google Meet. Los recursos Google Calendar y Google Doodle han sido utilizados para programar las sesiones. Además, se ha elaborado en Google Drive un repositorio donde se ha puesto a disposición de todos los participantes los recursos y materiales generados en el equipo docente, así como facilitar la colaboración y el trabajo en equipo. Por otro, se ha elaborado una página web en la plataforma Google Site en la que se han publicado las sesiones actualizadas y enlaces a los recursos generados.

Por último, se ha diseñado un cuestionario final en Google Formulario para valorar la satisfacción de los miembros del equipo docente a la finalización del mismo.

\section{Resultados}

En la sesión inicial se realizó la presentación del equipo docente y la dinámica a seguir durante el desarrollo del proyecto, así como las herramientas que se iban a utilizar distintas a las disponibles en Google Apps y que implicaban que cada componente del equipo creará una cuenta (por ejemplo Kahoot y Miro). 


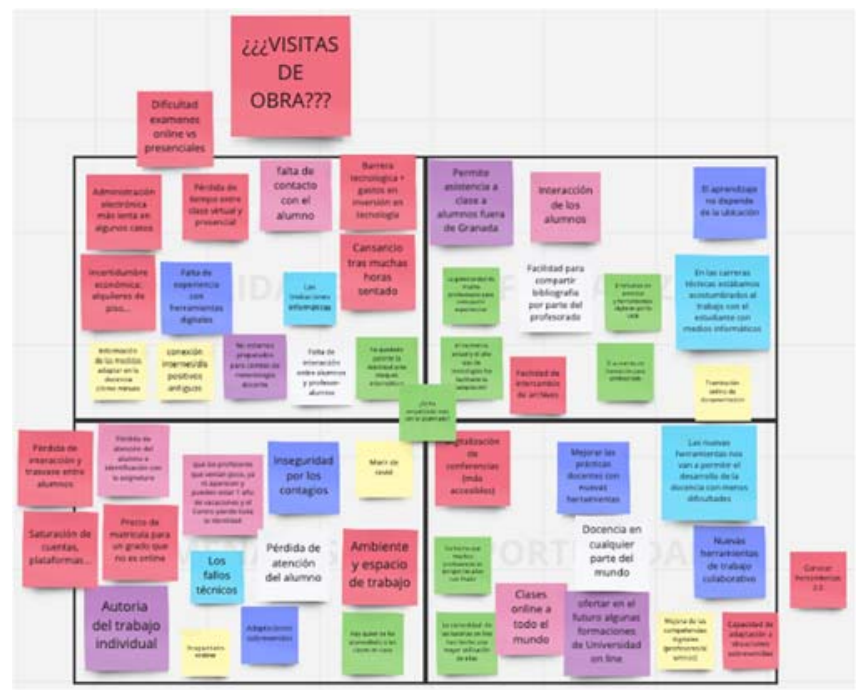

Fuente: Elaboración propia

Fig. 1 Matriz DAFO: docencia en la pandemia.

En la siguiente sesión de trabajo realizó una matriz DAFO sobre la adaptación de la docencia a un entorno online. Para ello se ha utilizado la pizarra colaborativa Miro (Fig. 1). En la Web creada con Google Site (Fig. 2) se fueron incorporando la infomación general e incluyendo la programación que estaba vinculada con un documento de Google Drive que se iba actualizado cada semana.

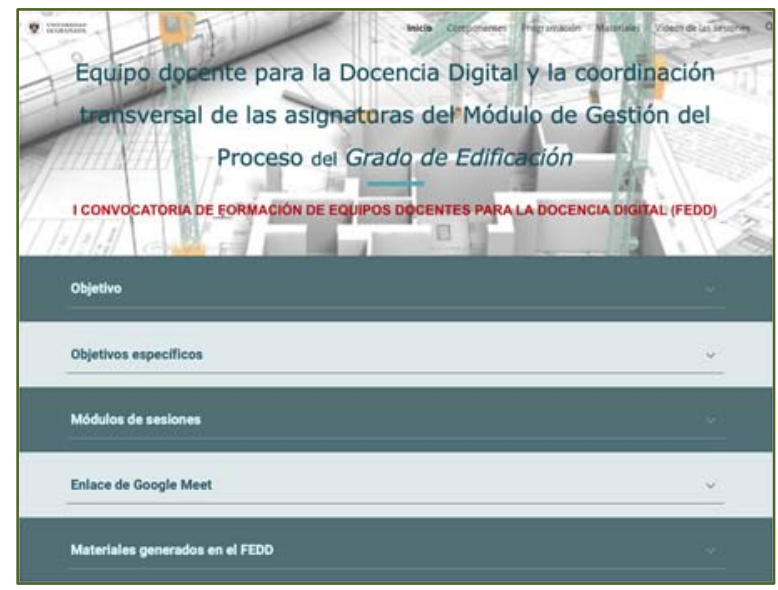

Fuente: Elaboración propia

Fig. 2 Web del Equipo docente. 


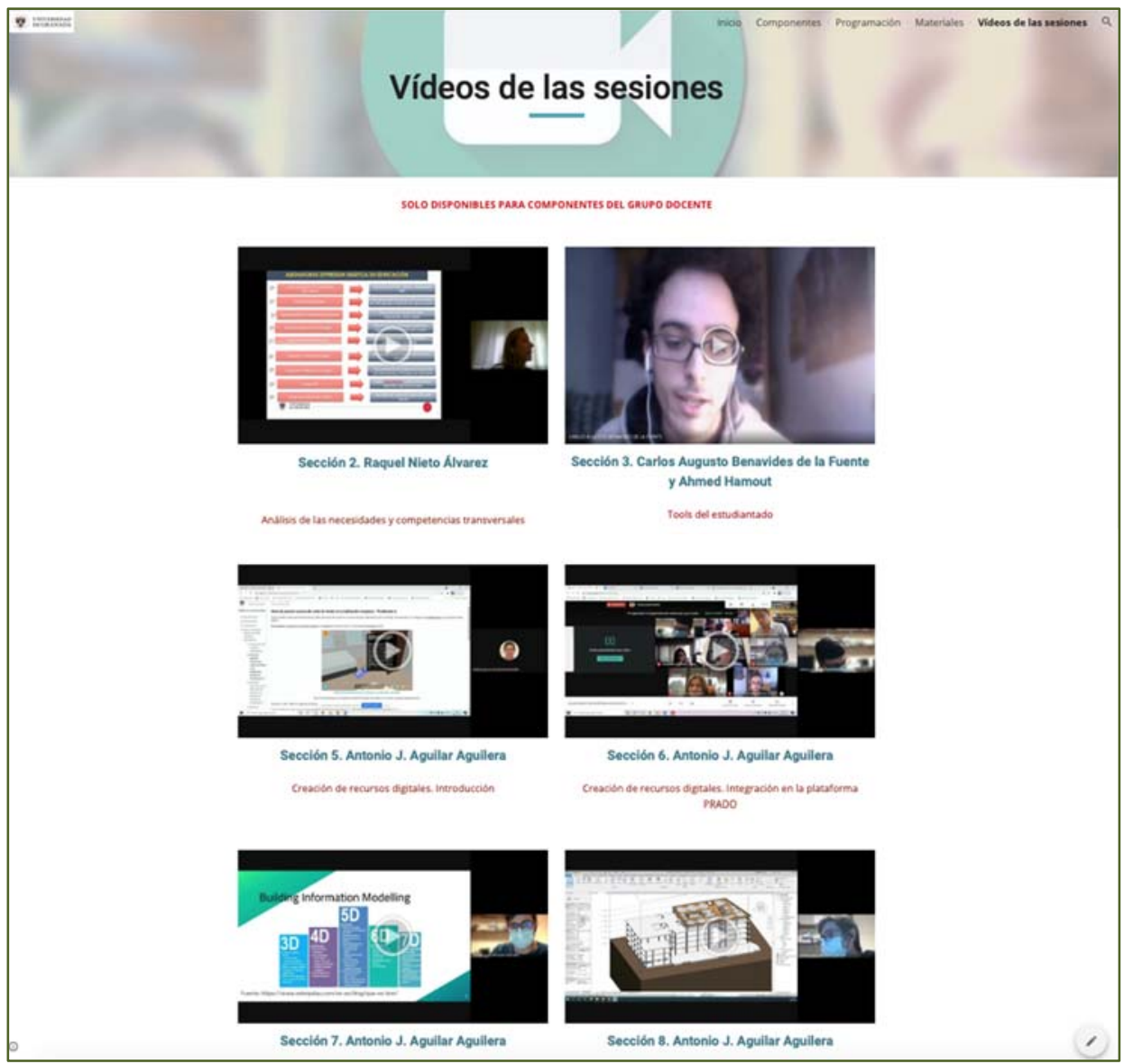

Fuente: Elaboración propia

Fig. 3 Vídeos de la sesiones disponibles en la Web.

También se añadieron los materiales utilizados o que eran necesarios para trabajar y/o seguir correctamente los contenidos. Dado que todas sesiones fueron grabadas, tras terminar las clases los vídeos estaban disponibles para faciliar la consulta de dudas sobre puntos concretos explicados (ver Fig. 3).

La metodología BIM ha tenido un gran peso en las sesiones desarrolladas en el marco del equipo docente. Las herramientas que proporciona esta metodología pueden utilizarse como un excelente recurso en el proceso de enseñanza-aprendizaje. Concretamente, en las sesiones se ha examinado el potencial uso de los modelos 3D BIM con el objetivo de analizar las características y configuraciones de las soluciones constructivas (ver Fig. 4) y la elaboración de entornos digitales interactivos que permitan su visualización mediante realidad virtual (ver Fig 5). 
Equipo docente para la Docencia Digital y la Coordinación Transversal de las asignaturas del Módulo de Gestión del Proceso del Grado de Edificación. Universidad de Granada - Teaching Team for Digital Teaching and Transversal Coordination of the subjects of the Process Management Module of the Degree in Building. University of Granada

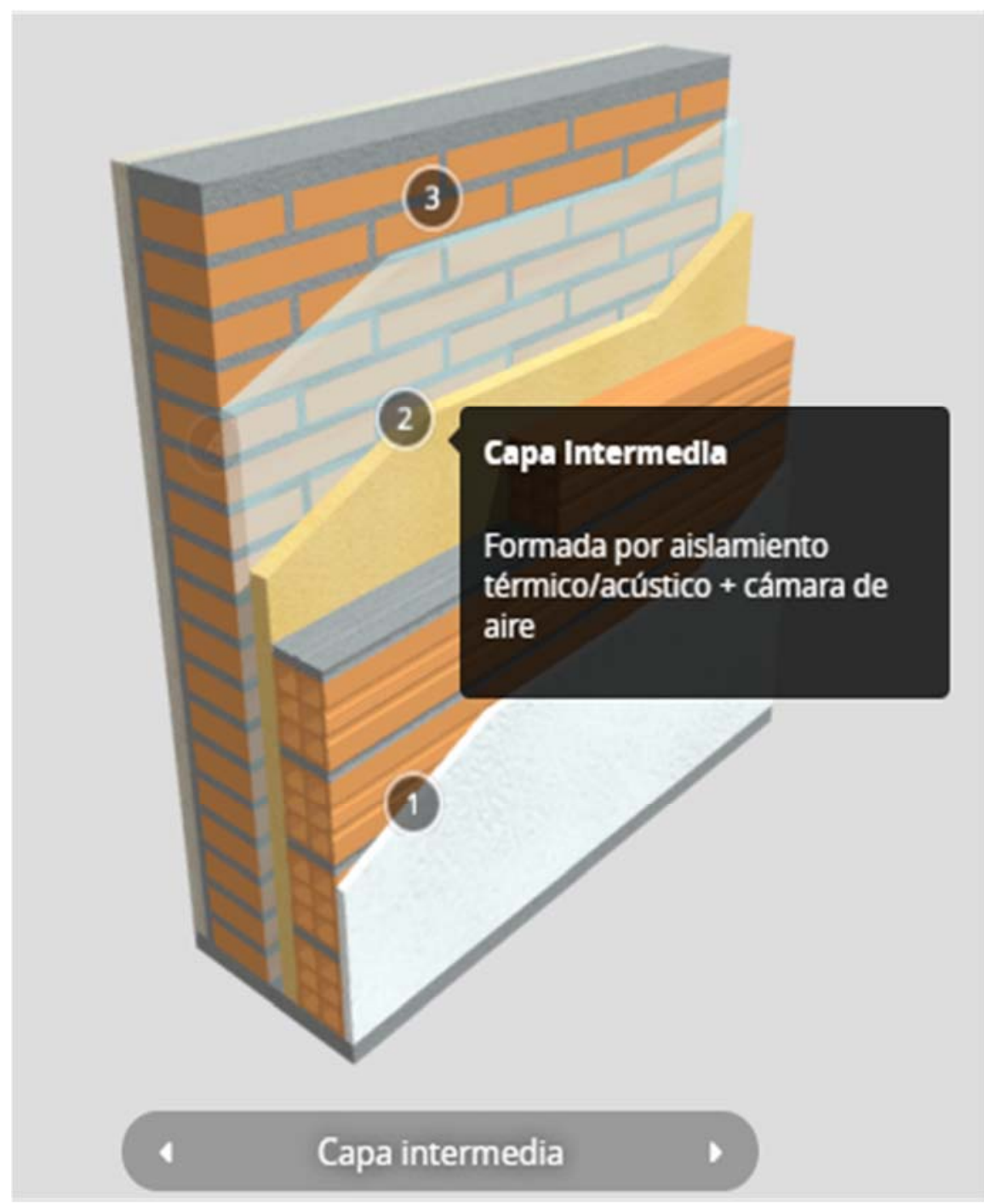

Fig. 4. Modelo digital de una solución constructiva tipo.

Fuente: Elaboración propia 
Antonio J. Aguilar, Carlos Benavides de la Fuente, Gloria Cuenca-Moyano, Lourdes Gutiérrez-Carrillo, María L. de la Hoz-Torres, María Martín-Morales, M. Dolores Martínez-Aires, Manuel Martínez Carrillo y Raquel Nieto-Álvarez

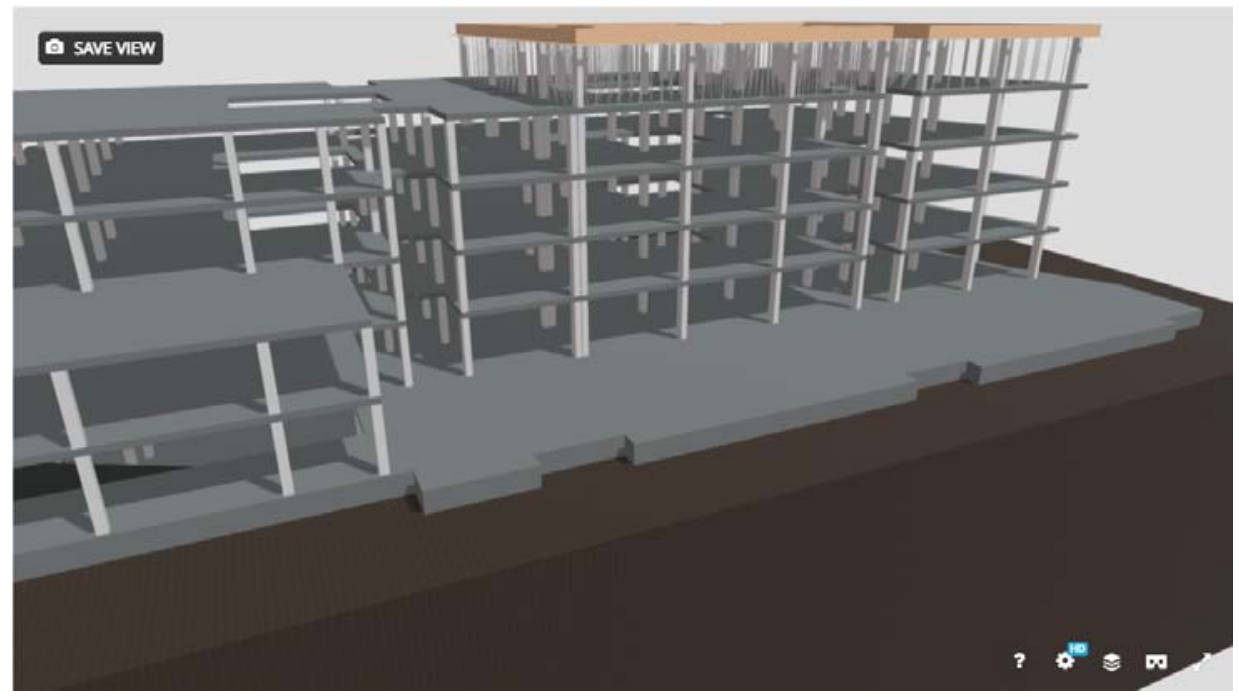

Fig. 5. Entorno de realidad virtual generado a partir de un modelo BIM.

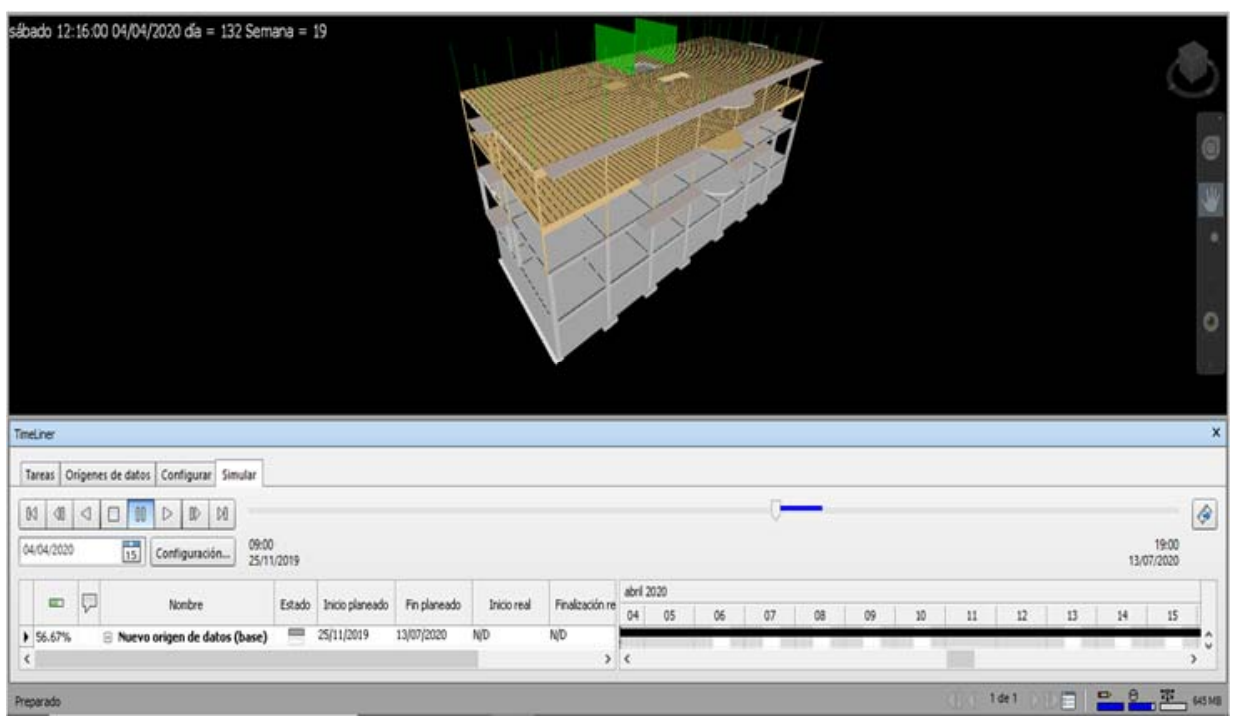

Fig. 6 Simulación de la planificación del proceso constructivo.

Fuente: Elaboración propia

Por otro lado, se profundizó en el uso de programas informáticos y herramientas BIM 4D para el análisis y gestión de la organización y programación de procesos de ejecución en obra, 
Equipo docente para la Docencia Digital y la Coordinación Transversal de las asignaturas del Módulo de Gestión del Proceso del Grado de Edificación. Universidad de Granada - Teaching Team for Digital

Teaching and Transversal Coordination of the subjects of the Process Management Module of the

Degree in Building. University of Granada

destacando su capacidad para facilitar la adquisición de conocimiento en relación a la interacción de los distintos procesos a partir de simulaciones (ver Fig. 6).

En este contexto, se realizó una introducción al uso de videos e imágenes $360^{\circ}$ para acercar al alumnado la experiencia de visitar una obra, facilitando la interacción con el contenido y pudiendo utilizarse para generar una experiencia inmersiva (ver Fig. 7). La incorporación de este tipo de recursos, unidos a los proporcionados por la metodología BIM, permiten la digitalización y creacción de nuevos recursos para la adquisición de competencias relacionadas con la docencia de las asignaturas del Módulo de Gestión del Proceso del Grado en Edificación.

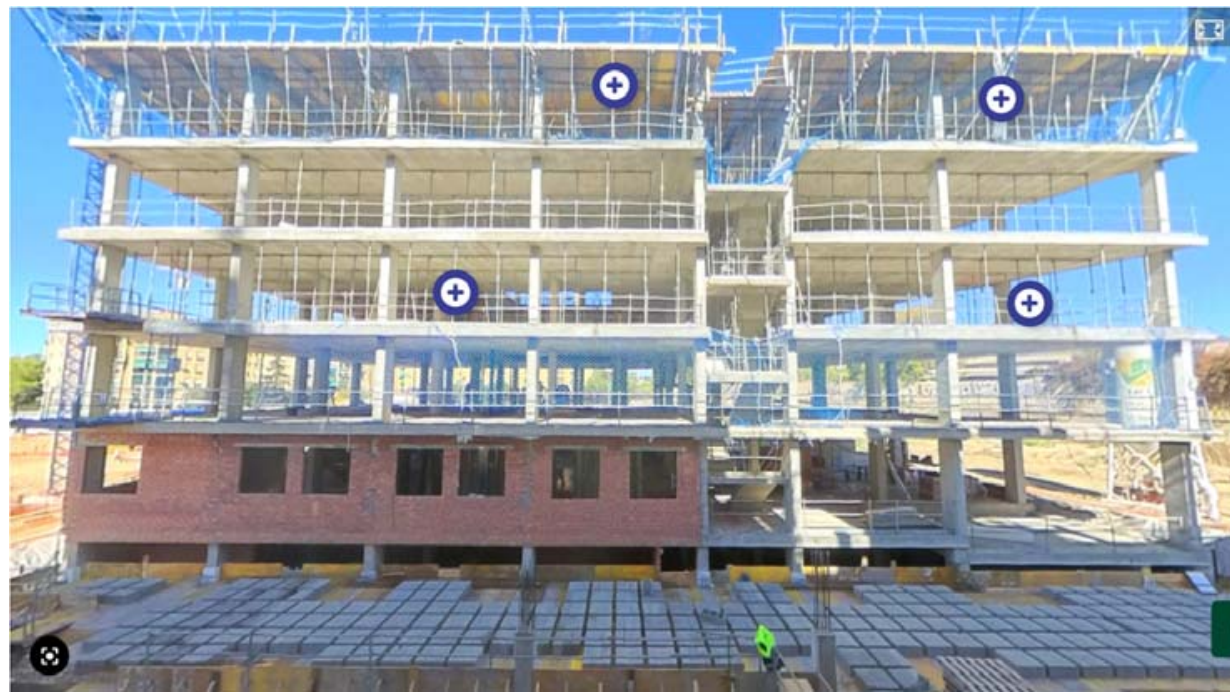

Fuente: Elaboración propia

Fig. 7. Imagen $360^{\circ}$ con contenido interactivo.

En la sesión final se ha realizado una Gráfica de Plus - Delta también utilizando la herramienta Miro. En esta gráfica en una columna se ha anotado lo positivo, o las cuestiones que se desarrollaron con un resultado positivo, y en la otra las áreas que necesitan mejorar (Ver Fig, 8).

Cabe destacar que uno de los puntos fuertes del equipo ha sido la participación de los estudiantes. Ellos han aportado un punto de vista enriquecedor y que como docentes es necesario conocer. 
Antonio J. Aguilar, Carlos Benavides de la Fuente, Gloria Cuenca-Moyano, Lourdes Gutiérrez-Carrillo, María L. de la Hoz-Torres, María Martín-Morales, M. Dolores Martínez-Aires, Manuel Martínez Carrillo y Raquel Nieto-Álvarez

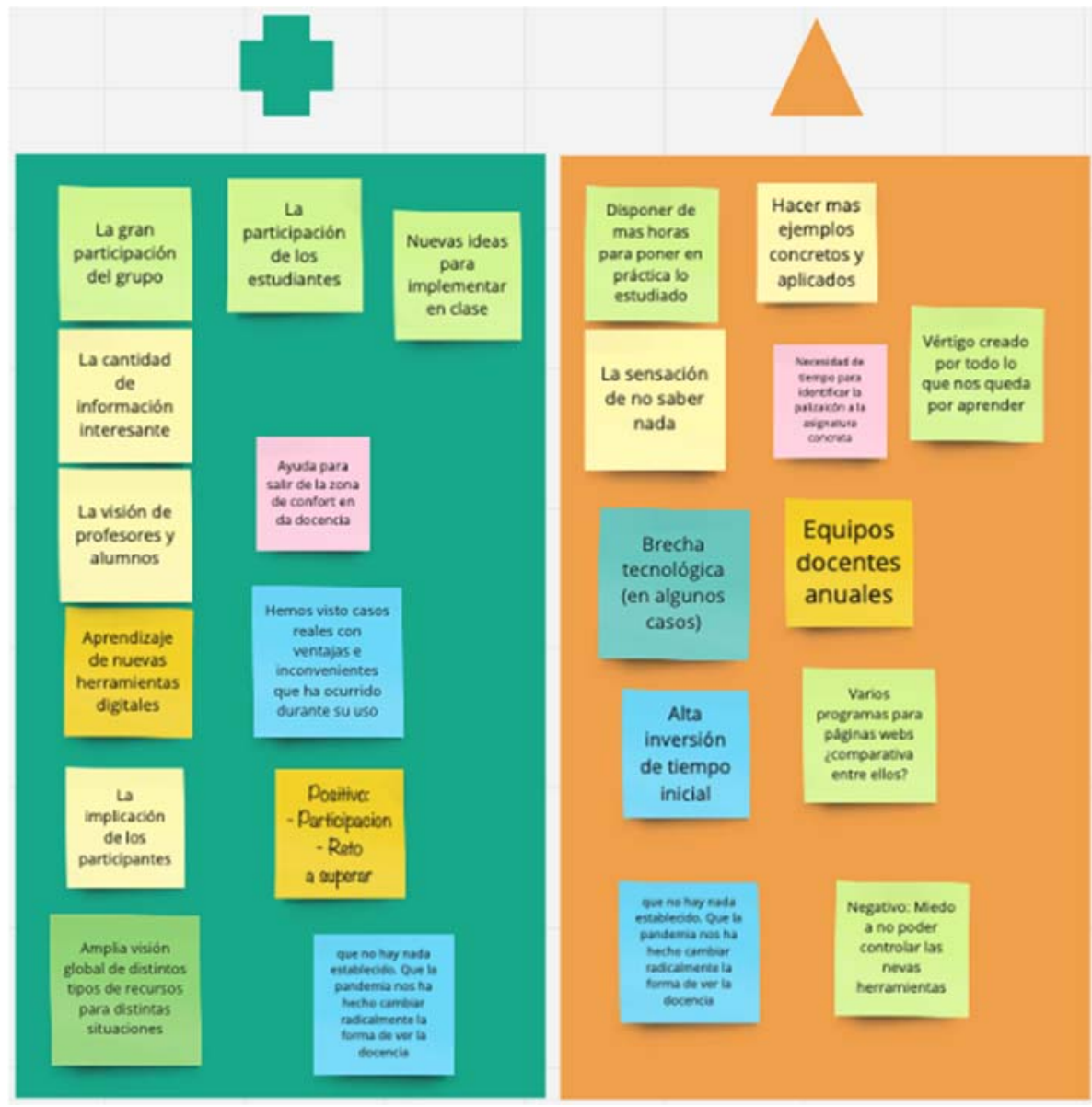

Fuente: Elaboración propia

Fig. 8 Gráfica de Plus - Delta.

Para cerrar la experiencia, se ha realizado la encuesta de satisfacción de valoración numérica de 1 a 5. Los resultados, recogidos en la Tabla 2, han dejado patente la satisfacción general con el equipo docente, así como la destacable participación. Así mismo, hay que destacar como resultado de la misma que, dada la gran cantidad de información y herramientas suministradas, se demanda una formación continuada que permite profundizar en su estudio y en el diseño de distinto material curricular para las asignaturas. 
Tabla 2. Resultado de la encuesta final

\begin{tabular}{|l|c|}
\hline \multicolumn{1}{|c|}{ Item } & Valoración \\
\hline INFORMACIÓN RELATIVA A LA ACCIÓN FORMATIVA & \\
\hline Los objetivos de la acción formativa han sido & 5 \\
\hline formulados de manera clara y concreta & 4,8 \\
\hline adecuados a las prácticas desarrolladas en el aula & 4,7 \\
\hline \begin{tabular}{l} 
Los contenidos de la acción formativa \\
\hline han sido claros y ha existido un orden lógico en su exposición
\end{tabular} & 4,8 \\
\hline han permitido desarrollar los temas tratados con un nivel adecuado & 4,9 \\
\hline La metodología & 4,7 \\
\hline ha favorecido la motivación y participación & 5 \\
\hline ha favorecido el desarrollo de actividades grupales & 4,9 \\
\hline Las actividades realizadas & \\
\hline han repercutido en mejorar mi conocimiento sobre la temática & 4,8 \\
\hline han sido las adecuadas para mejorar mis destrezas docentes & 4,8 \\
\hline La organización temporal & \\
\hline ha facilitado que las tareas teóricas y prácticas se realicen en los momentos \\
\hline adecuados & 4,9 \\
\hline ha facilitado el desarrollo total del curso & 4,9 \\
\hline La información facilitada ha sido adecuada respecto a & \\
\hline la posibilidad de aplicación de los conocimientos a la práctica docente & 4,8 \\
\hline lo necesario para hacer un seguimiento del curso & \\
\hline REFLEXIÓN PERSONAL SOBRE LA ACCIÓN FORMATIVA & \\
\hline $\begin{array}{l}\text { Mi implicación en la acción formativa como alumnado ha sido adecuada en } \\
\text { cuanto a }\end{array}$ & \\
\hline asistencia & \\
\hline participación activa & \\
\hline
\end{tabular}

\section{Conclusiones}

Dentro de los Planes de Formación e Innovación Docente de la UGR, los equipos docentes son una buena herramienta de formación, de colaboración e intercambio de experiencias entre profesorado experto y principiante con el objetivo de la mejora continua de la práctica docente.

La pandemia ha demandado una rápida adaptación del profesorado y de las metodologías docentes a la enseñanza online, lo que ha hecho necesario reforzar la formación en herramientas digitales. Con este objetivo, la UGR ha fomentado la creación de Equipos Docentes para la docencia digital. En este contexto surge el Equipo docente para la Docencia 
Antonio J. Aguilar, Carlos Benavides de la Fuente, Gloria Cuenca-Moyano, Lourdes Gutiérrez-Carrillo, María L. de la Hoz-Torres, María Martín-Morales, M. Dolores Martínez-Aires, Manuel Martínez Carrillo y Raquel Nieto-Álvarez

Digital y la Coordinación Transversal de las asignaturas del Módulo de Gestión del Proceso del Grado de Edificación. Su objetivo principal ha sido contribuir al desarrollo de competencias docentes digitales particularmente en el profesorado del Dpto. de Construcciones Arquitectónicas con docencia en las asignaturas del Módulo de Gestión de Proyectos y, de forma general, del Grado en Edificación.

A lo largo de las sesiones programadas, quienes integraban el grupo han ido conociendo las posibilidades de herramientas de creación de recursos digitales interactivos, el uso de la metodología Building Information Modelling (BIM), de la realidad virtual y videos $360^{\circ}$, que pueden acercar al alumnado a las obras y profundizar en conceptos específicos del grado.

Además, se han incorporado diferentes sesiones para mostrar herramientas de asistencia activa, tan necesaria en la situación de la pandemia, pero que pueden utilizarse en la docencia presencial.

Durante las primeras sesiones se ha comprobado que otro de los puntos fuertes de de la propusta ha sido contar en el equipo con dos estudiantes. Ellos han aportado una visión distinta de la adaptación de la docencia provocada por la crisis sanitaria de la COVID-19. La sesión que han impartido, así como su activa participación, han enriquecido los objetivos inicialmente planteados.

Este primer contacto ha permitido un acercamiento a una gran cantidad de contenidos desconocidos para la mayoría del profesorado que integra el equipo. Los resultados globales de la experiencia han sido muy positivos y enriquecedores, lo que se ha ido reflejando en una asistencia continuada de quienes integran el grupo docente así como por una insistente demanda de proseguir con una formación continua en estas herramientas avanzadas.

\section{Referencias}

CEPRUD (2021). Centro de Producción de Recursos para la Universidad Digital de la Universdiad de Granada <https://ceprud.ugr.es> [Consulta: 15 de junio de 2021].

UGR (2016). Planes de formación docente de la Universidad de Granada. $<$ https://calidad.ugr.es/areas/formacion-innovacion-docente/fomacion/cursos/acciones-formativas18-20> [Consulta: 15 de junio 2021].

UGR 2020a (2020). Plan de actuación COVID-19 de la Universidad de Granada. <https://covid19.ugr.es/informacion/docencia-virtual> [Consulta: 16 de junio 2021].

UGR 2020b (2020). Formación de Equipos para la Docencia Digital de la Universidad de Granada. $<$ https://calidad.ugr.es/areas/formacion-innovacion-docente/formacion/docencia-digital> [Consulta: 15 de junio 2021].

Sarkodie, S. A., y Owusu, P. A. (2021). "Global assessment of environment, health and economic impact of the novel coronavirus (COVID-19)" en Environment, Development and Sustainability, 23, p. 50055015 . 